\title{
Resolution of an external layer macular hole associated with an optic nerve pit after laser photocoagulation
}

\author{
Philip M Falcone, Peter L Lou
}

Optic nerve pits are estimated to occur in 1 in 11000 patients. ${ }^{12}$ Maculopathy associated with optic nerve pits occurs in $30 \%-63 \%^{23}$ and is characteristically described as a serous retinal detachment. Brown et $\mathrm{al}^{3}$ reviewed 75 patients with optic nerve pits and found that 24 of the 38 patients with macular detachments had cystic changes in the macula. Lamellar macular holes were present in the outer layer of the retina in 10 of the 24 eyes.

Lincoff $e t$ al noted an associated retinoschisis in patients with optic pits. A macular hole was observed in the outer retinal layer in 14 out of the 15 eyes examined and was associated with severely diminished visual acuity.

We now present a case of an optic nerve pit with retinoschisis and a outer layer detachment with an external layer macular hole that was treated promptly with krypton red barrier photocoagulation. The marked visual improvement seen in our patient corresponded to the resolution of the external layer macular hole observed clinically and on fluorescein angiography.

\section{Case report}

In February 1989, a 45-year-old woman was referred after complaining of blurred vision in her left eye over a 1 week period. She had no significant medical or ocular history. She reported to have enjoyed excellent vision in both eyes throughout her life. The examination showed best corrected visual acuities to be 20/20 in the right eye and counting fingers at 6 feet in the left eye. Amsler grid testing revealed a central scotoma in the left eye. External and slitlamp examination were unremarkable. The right fundus appeared normal. The left optic nerve

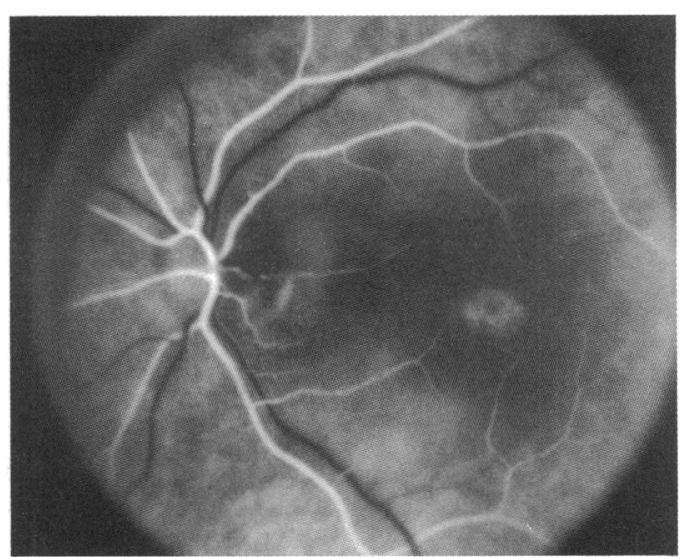

Figure 2 Early frame fluorescein angiography discloses hypofluorescence of the optic nerve pit with an RPE transmission defect in the fovea.

had a $0.2 \mathrm{cup} / \mathrm{disc}$ ratio with a optic pit at the temporal border. A schisis-like elevation of the retina was observed extending temporally from the optic pit to the macula area. A localised serous retina detachment of the macula was present but did not appear to communicate with the disc. At the fovea, a $700 \mu \mathrm{m}$ external layer hole was present. The external layer hole had scalloped edges which turned inward towards the overlying intact retinal layers (Fig 1). Fluorescein angiography revealed early hypofluorescence of the temporal margin of the disc with late leakage, consistent with the diagnosis of an optic nerve pit. A retinal pigment epithelium (RPE) window defect was present in the macula corresponding to the external layer observed clinically (Figs 2 and 3). Testing with laser scotometery ${ }^{4}$ revealed an absolute scotoma using $50 \mu \mathrm{m}$ spot size at the highest intensity.

Given the poor visual acuity and macular

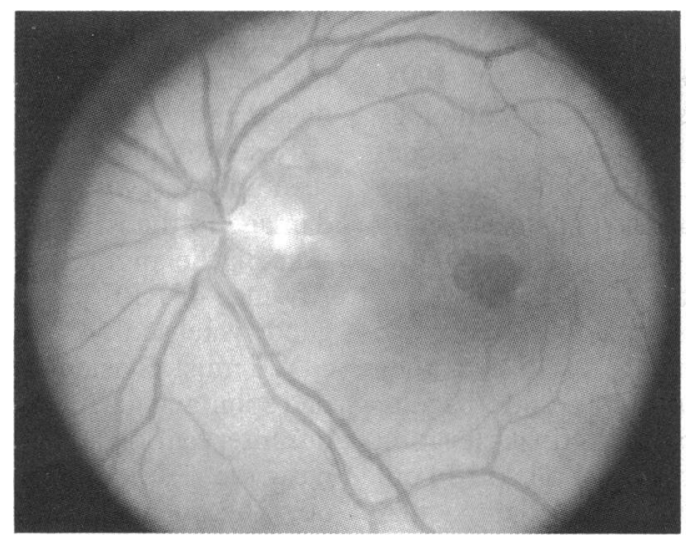

Figure 1 Optic nerve pit with peripapillary retinoschisis. A serous detachment of the macula is present with a $700 \mu \mathrm{m}$ external layer hole.

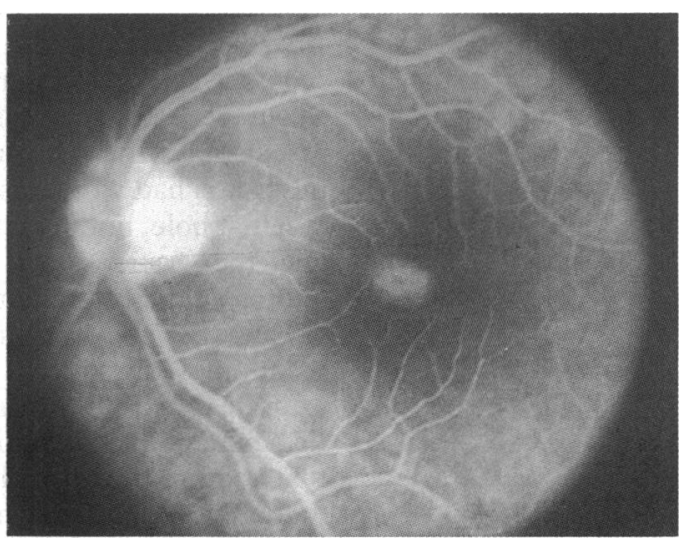

Figure 3 Late frame fluorescein angiography reveals leakage from the optic nerve pit. Note the intact inner retinal layer overlying the lamellar macular hole. 


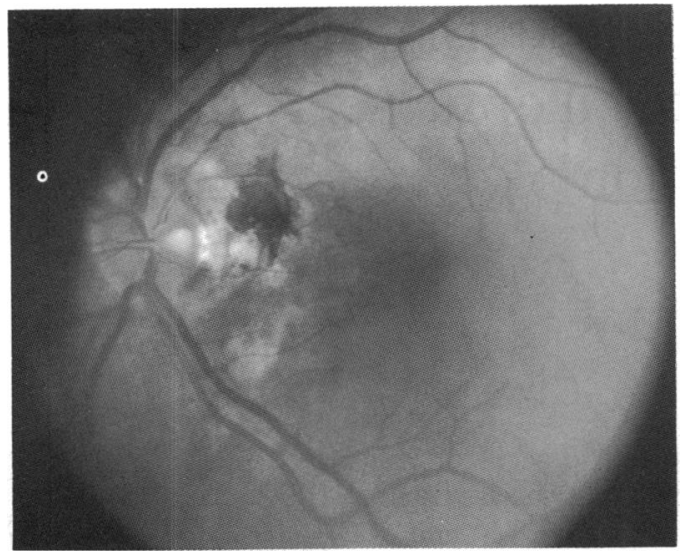

Figure 4 After treatment, the retina has reattached with peripapillary RPE change. Mild pigment mottling is seen in the otherwise normal appearing macula.

change, treatment was initiated with krypton red photocoagulation. Using $200 \mu \mathrm{m}$ spot size, two rows of laser were placed concentric to the disc in a barrier fashion previously described ${ }^{56}$ from 12 o'clock clockwise to 6 o'clock extending into attached retina both above and below the disc.

The patient returned for follow up examination in March 1989. The vision in her left eye improved to 20/160. Laser scars were evident in the peripapillary retina but there was otherwise negligible change in the appearance of the macula. Additional photocoagulation was applied in a row temporal to the area previously treated.

In April 1989, 6 weeks after her initial treatment, the patient reported improved vision. On examination, visual acuity in the left eye was measured at 20/60. The schisis-like change surrounding the disc had resolved along with the external layer detachment and external layer hole. The central fovea exhibited pigment mottling (Fig 4). Fluorescein angiography in the macular region appeared normal (Fig 5).

The patient has been followed for 2 years and the retina has remained attached. At the last follow up examination, the vision in the left eye was $20 / 40$. The patient reported a small superotemporal scotoma on Amsler grid testing and did not note any scotoma upon testing with laser scotometry.

\section{Comment}

Macular holes complicating optic pit detachments were first described by Reis ${ }^{1}$ in 1908. Sugar ${ }^{7}$ reported nine cases of macular pathology associated with optic nerve pits. One patient described had what was initially believed to be a macular hole, but the return of good visual acuity and the cystic retinal appearance led to the author's conclusion that this was a 'false hole'.

Lincoff and colleagues, ${ }^{4}$ using stereoscopic techniques, discovered that 13 of 15 eyes they examined with optic nerve pits and macular detachments had a schisis-like retinal elevation that communicated with the optic pit and 14 out of the 15 eyes had external layer lamellar macular holes. The internal limiting membrane remained intact in every patient and none of the holes reached full thickness.

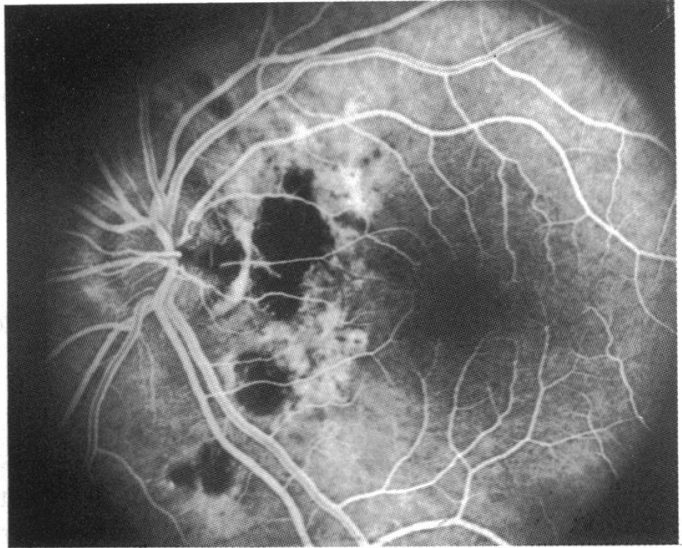

Figure 5 Mid-venous phase of the fluorescein angiogram. There is resolution of the RPE window defect.

$\mathrm{Cox}^{8}$ reviewed various therapeutic modalities in the treatment of macular detachments with optic nerve pits. Only two patients were described with symptomatology of 4 months or less in duration. Both patients regained better than 20/ 50 vision after prompt intervention combining pars plana vitrectomy with gas tamponade and laser photocoagulation.

In our patient treatment with laser photocoagulation alone resulted in reattachment of the retina with resolution of both the macular detachment and presumed external layer macular hole. The markedly improved visual acuity may be due to either of these events independently or in association. However, the absence of the previously noted central scotoma on both laser scotometry and Amsler grid testing confirms that the macular hole did in fact resolve.

Histopathologically, a macular hole represents an area devoid of photoreceptors. ${ }^{9}$ Since closure of the hole may cause reabsorption of surrounding subretinal fluid and oedema, this may result in return of good vision assuming the neighbouring photoreceptors are functioning. ${ }^{10}$

Current treatment recommendations for treating an optic nerve pit-related sensory retinal detachment suggest observation for 3 months unless there is macular pathology (such as foveal cyst or window defect). ${ }^{6}$ Our patient was treated after 1 week of pronounced visual loss. The recovery of good visual acuity with evidence of resolution of the external layer macular hole after prompt laser photocoagulation supports the premise that early reattachment of the retina may play a role in preventing photoreceptor degeneration. ${ }^{11}$

Resolution of the RPE transmission defect seen on fluorescein angiogram suggests that the damage to the RPE was temporary or regeneration of the pigment epithelial layer occurred. Absence of RPE presumably does not preclude good visual acuity. ${ }^{12}$

We must consider that our patient may have had spontaneous reattachment of the macula and our intervention had little to do with the eventual outcome. Although this event cannot be ruled out, the poor natural course in the majority of optic pit detachments with the marked anatomical and clinical improvement in our patient within 6 weeks of initial laser surgery lead 
us to believe that there was indeed a treatment effect.

Since reattachment of the retina and resolution of the macular hole was achieved solely with laser photocoagulation, we believe this should be the initial procedure in the management of these patients.

1 Reis W. Eine wenig bekannte typische missbildung am sehnerveneintritt: umnschiebene grubenbildung auf papilla n optici. Zeitschr Augenheilkd 1908; 19: 505-28.

$2 \mathrm{Kranenburg} \mathrm{EW}$. Crater-like holes in the optic disc and central serous retinopathy. Arch Ophthalmol 1960; 64: 912-28.

3 Brown GC, Shields JA, Goldberg RE. Congenital pits of the optic nerve head. II. Am F Ophthalmol 1980; 87: 51-65.

4 Lincoff H, Lopez R, Kreissig I, Yannuzz I, Cox M, Burton T Retinoschisis associated with optic nerve pits. Arch Ophthalmol 1988; 106: 61-7.
5 Brockhurst RJ. Optic pits and posterior retinal detachment. Trans Am Ophthalmol Soc 1975; 73: 264-88.

6 Schatz H, McDonald HR. Treatment of sensory retinal detachment associated with optic nerve pit or coloboma. Ophthalmology 1988; 95: 178-86.

7 Sugar HS. Congenital pits in the optic disc and their equivalents (congenital coloboma and colobomalike excavations) associated with submacular fluid. Am $\mathcal{F}$ Ophthalmol 1967; associated with submacular fluid. Am $\mathcal{F}$ Ophthalmol 1967;

8 Cox MS, Witherspoon CD, Morris RE, Flynn HW. Evolving
techniques in the treatment of macular detachment caused by optic nerve pits. Ophthalmology 1988; 95: 889-96.

9 Frangieh GT, Green WR, Engle HM. A histopathologic study of macula cysts and holes. Retina 1981; 1:311-36.

10 Fine SL. Vitreous surgery for macula hole in perspective. Is there an indication? Arch Ophthalmol 1991; 109: 635-6.

11 Kelly NE, Wendell RT. Vitreous surgery for idiopathic macular holes. Results of a pilot study. Arch Ophthalmol 1991; 109: 654-9.

12 Bressler NM, Finkelstein D, Sunness JS, Maguire AM, Yarian D. Retinal pigment epithelial tears through the fovea with preservation of good visual acuity. Arch Ophthalmol 1990; 108: 1694-7.

\section{A case of presumed congenital herpes zoster ophthalmicus}

\author{
J Singh, J M Gibson
}

Herpes zoster ophthalmicus is a disease occurring in the elderly but it only rarely occurs in children. We report an unusual case of presumed herpes zoster ophthalmicus occurring in a newborn infant. It is postulated that maternal infection with chicken pox during the second trimester of pregnancy has led to in utero transmission of the varicella virus resulting in presumed congenital herpes zoster ophthalmicus. This is the first report of such a case to our knowledge.

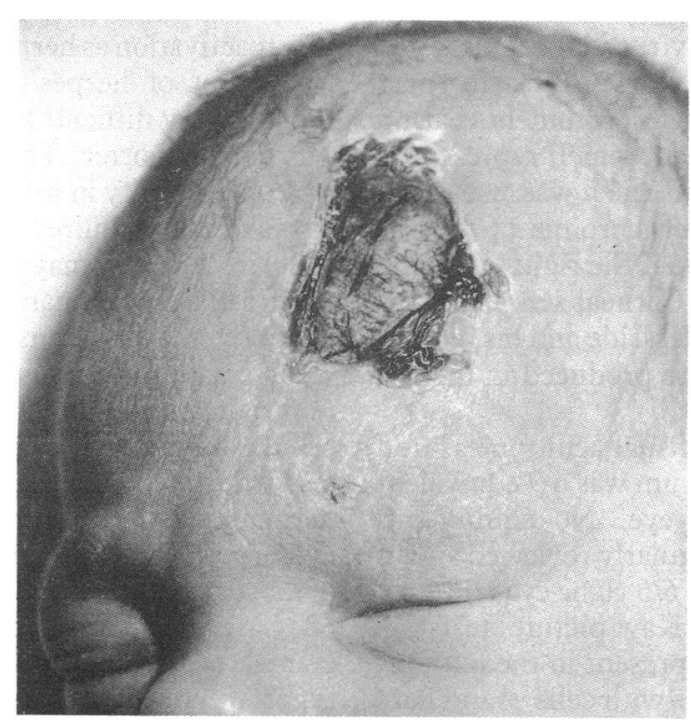

Figure 1 Newborn infant. A full thickness skin defect is seen in the ophthalmic division of the trigeminal nerve.

\section{Case report}

A mother who contracted chicken pox at the beginning of the second trimester of pregnancy, gave birth to a 7 week preterm infant boy, weighing $2 \cdot 1 \mathrm{~kg}$. Maternal chicken pox infection was confirmed by the presence of a typical generalised rash in association with significantly raised IgM titres suggesting a recent infection.

At birth it was found that the boy had a large full thickness skin defect exposing the skull bones in the distribution of the ophthalmic

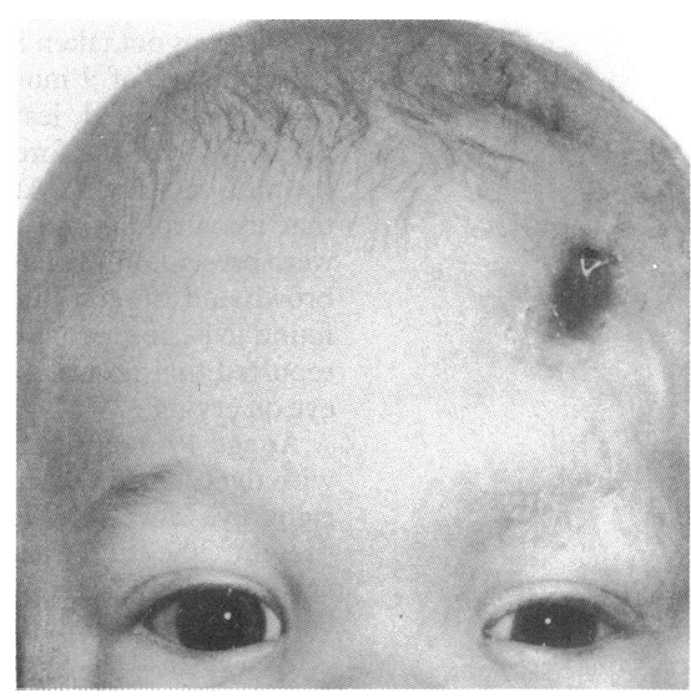

Figure 2 Aged 5 months. Healing skin defect. The extent of the original affected area of skin can be seen from the surrounding scarring. It respects the mid-line. 